\title{
Rückwirkungen der \\ Binnenmarktfreiheiten auf Gesundheitsmärkte
}

\section{Chancen und Risiken für Deutschland und Polen ${ }^{1}$}

Gesundheitsmärkte sind gleichzeitig durch Globalisierung und Lokalisierung geprägt. Sie sind stark national reguliert, aber sie unterliegen auch den Regeln des europäischen Binnenmarktes. Daraus ergeben sich Risiken und Chancen für deutsche und polnische Gesundheitsmärkte. Bei Mobilität auf dem Arbeitsmarkt wird wegen des Einkommensgefälles eine Zuwanderung nach Deutschland erfolgen, wohingegen die Inanspruchnahme medizinischer Dienstleistungen und Kuren von Deutschen in Polen ein sehr begrenzter Markt bleiben wird.

\section{Leonhard Hajen}

\section{Globalisierung und Lokalisierung}

Aus der Sicht der Regionen in der Europäischen Union bedeutet die Globalisierung von Märkten eine Chance, eigene Produkte weltweit abzusetzen, aber auch ein Risiko, im weltweiten Handel nicht mithalten zu können, weil die Produktivität zu niedrig ist und/oder die Arbeitslöhne und Steuern zu hoch sind. Aus der Sicht Westeuropas haben Unternehmen in Mittel- und Osteuropa eine Reihe Standortvorteile. Gemeinsam sieht sich Europa der Konkurrenz mit Produkten aus Asien konfrontiert. Sowohl aus der Sicht der ökonomischen Theorie als auch der historischen Erfahrung mehrt freier Handel den Wohlstand, aber Vor- und Nachteile sind regional sehr unterschiedlich verteilt und damit können die Auswirkungen auf die Beschäftigung für einzelne Regionen durchaus dramatisch sein. Die Stichworte Schiffbau und Textilindustrie mögen hier als Beispiele genügen, wie international gehandelte Güter zu regional hoher Arbeitslosigkeit führen können.

Umgekehrt versprechen sich Regionen, die traditionell eine starke Stellung in der Bereitstellung von Gesundheitsgütern haben, Wachstums- und Beschäftigungschancen. Kur- und Heilbäder haben aufgrund natürlicher Ressourcen wie Luft und Wasser mit besonderer Heilwirkung immer schon Gäste aus dem Ausland gehabt. In der Grenzregion zwischen Polen und Deutschland könnte sich auch ein gemeinsamer Gesundheitsmarkt entwickeln, von dem Patienten und Ärzte und andere medizinische Berufe auf beiden Seiten der Grenze profitieren. Gesundheitsdienstleistungen zur Heilung akuter Krankheiten, aber auch Prävention und Rehabilitation gehören zu den Wachstumsmärkten der Zukunft. Andererseits zeigt sich schon heute, dass neben diesen Chancen zusätzlicher Beschäf-

Prof. Dr. Leonhard Hajen, Universität Hamburg, Department für Wirtschaft und Politik tigung für spezielle Regionen auch Risiken erkennbar sind, die wiederum regional ungleich wirken. Ärzte und Pflegekräfte im Gesundheitssektor verfügen über eine hohe Qualifikation, die ihnen eine relativ problemlose Mobilität zwischen nationalen Arbeitsmärkten ermöglicht. Meine Hypothese ist, dass die Chancen, bei Kuren und Rehabilitationsmaßnahmen auf internationalen Märkten erfolgreich zu sein, relativ gering sind, aber eine Abwanderung von Ärzten und Pflegekräften aus Polen erfolgen wird, wenn die Einkommensdifferenzen zwischen Deutschland und Polen hoch bleiben. Um die Aussage zu überprüfen, sind die besonderen Eigenschaften von Gesundheitsmärkten zu analysieren, die sie von sonstigen Dienstleistungsmärkten unterscheiden. Gleichzeitig ist der Gesundheitssektor ein Markt, der dem europäischen Wettbewerbsrecht unterliegt. Dies setzt den nationalen Gestaltungsspielräume bei der Finanzierung und Bereitstellung von Gesundheitsgütern Grenzen, die für den Wettbewerb und die Chancen und Risiken von Regionen relevant sind.

Gesundheitsmärkte sind ein gutes Beispiel, dass gleichzeitig ein Prozess der Globalisierung und der Lokalisierung stattfindet. Medikamente und medizinische Geräte werden international gehandelt, aber der größere Teil der Umsätze sind medizinische Dienstleistungen, die „faceto-face" erbracht werden, also der Ort der Produktion und des Verbrauchs sind identisch. Es sind im Wesentlichen regional zu erbringende Dienstleistungen. Globalisierung ist kein linearer Prozess, sondern neben der Globalisierung gibt es gleichzeitig eine Tendenz der Lokalisierung, weil ein wachsender Anteil der Produktion nicht international gehandelt wird. Das Volumen der international gehandelten Güter gemessen an der Summe von Exporten und Importen wächst, aber ihr wertmäßiger Anteil an der Gesamtproduktion sinkt, weil international han- 
delbare Güter auf einem höheren Produktivitätsniveau erstellt werden und ihre Preise im Verhältnis zu Dienstleistungen sinken. ${ }^{2}$

\section{Besonderheiten medizinischer Güter}

Medizinische Dienstleistungen sind typische Beispiele für Güter, die aus „technischen“ Gründen regional produziert und konsumiert werden müssen. Märkte für Gesundheitsdienstleistungen gehören zudem zu den zukünftigen Wachstumsbereichen, weil einerseits die Möglichkeiten der Diagnose und Therapie aufgrund des medizinischen Fortschritts zunehmen, andererseits aber der Anteil älterer Menschen in der Bevölkerung zunimmt. ${ }^{3}$ Die Auswirkungen auf die Ausgaben für Gesundheit werden in der wissenschaftlichen Literatur streitig diskutiert, weil höheres Lebensalter nicht zwingend eine größere Morbidität bedeutet, sondern lediglich eine zeitliche Verlagerung hoher Behandlungskosten zur Folge haben könnte. ${ }^{4}$ Aber es gibt doch eine starke Tendenz, dass die Ausgaben für die Behandlung der Volkskrankheiten zunehmen und mehr Menschen professionelle Pflege nachfragen werden, so dass im Medizinsektor mehr Menschen Beschäftigung finden, weil gleichzeitig die Möglichkeiten der Rationalisierung in diesem Bereich gering sind. Weitere Expansionsmöglichkeiten werden daraus erwachsen, dass die Nachfrage nach Gesundheitsdienstleistungen im Bereich der Prävention mit einem fließenden Übergang zu Wellness-Angeboten zunehmen wird, die allerdings vollständig privat zu finanzieren ist und nicht zu den Leistungen gehört, die von einer gesetzlichen Krankenversicherung gedeckt werden. Beide Entwicklungen, also Gesundheitsmärkte im engeren, medizinischen Sinne als auch die „Wellness-Industrie“ können zusätzliche Perspektiven für eine regionale Entwicklung bieten, aber weil es sich um einen Prozess der Lokalisierung handelt, sind die Perspektiven gering, dass polnische oder deutsche Regionen einen Teil der internationalen Nachfrage in die eigene Region lenken.

Nicht alle Kurorte haben traditionell ein internationales Profil, wie z. B. Karlsbad oder Marienbad. Wie bei anderen Marktgütern sind auch bei Gesundheitsdienstleistungen die Qualität und der Preis zentrale Entscheidungsfaktoren. Ohne garantierte Behandlungsqualität wird künftig kein Leistungsanbieter wettbewerbsfähig sein. ${ }^{5}$ Aber selbst bei gleicher Qualität spielt bei Gesundheitsgütern der Preis eine geringere Rolle als bei „normalen“ Marktgütern. Gesundheitsgüter sind keine normalen Marktgüter, sondern sie sind „Vertrauensgüter“, d.h. im Unterschied zu normalen Gütern fehlt in der Regel ein Lernprozess, ob die gekauften Güter die gewünschte oder angemessene Qualität haben. ${ }^{6}$ Der Markt ist ein Prozess von „Versuch und Irrtum“ und aus der Vielzahl der Erfahrungen von Käufern und Verkäufern bildet sich Angebot und Nachfrage. Als Patient kann man weder beurteilen, ob der Krankheitsverlauf bei anderer Behandlung anders verlaufen wäre, noch ist es in der Regel möglich, sich aus einer Vielzahl von Kaufentscheidungen ein Urteil zu bilden und seine Nachfrage danach auszurichten. Medizinische Leistungen basieren auf Vertrauen der Nachfrager, dass die Leistungserbringer nicht aus Eigennutz, sondern im Interesse der Patienten handeln. Qualität und Preis der Behandlung werden je nach Ausgestaltung des nationalen Gesundheitssystems durch Gesetz, Standesrecht oder ärztliche Ethik gewährleistet, insofern sind es hoch regulierte Märkte, die mit Märkten im üblichen Sinne nur den Namen gemeinsam haben. Gesundheitsleistungen werden deshalb auch nur im Ausnahmefall über Marktpreise finanziert, sondern überwiegend durch Steuern (in staatlichen Gesundheitssystemen) oder Beiträgen der Versicherten (in Staaten mit gesetzlicher Krankenversicherung) bzw. Mischformen. Gesundheitsdienstleistungen sind aber auch in anderer Hinsicht Vertrauensgüter: Das Verhältnis zwischen Arzt und Patient spielt eine herausragende Rolle. Der Arzt als Anbieter entscheidet über die Nachfrage, also welche Behandlung der Patient bekommt, was der Grundannahme funktionsfähiger Märkte widerspricht, bei denen die Unanhängigkeit von Angebot und Nachfrage eine zentrale Prämisse ist. In dem Verhältnis zwischen Arzt und Patient spielt die Kommunikation in der Muttersprache und die Nähe zum Wohnort eine große Rolle, was die Möglichkeiten von Regionen, zu überregional oder sogar international nachgefragten Leistungsanbietern $\mathrm{zu}$ werden, erheblich eingrenzt. ${ }^{7}$ Trotz dieser Besonderheiten von Gesundheitsmärkten sind sie doch Märkte und die nationale Regulierung darf nicht im Widerspruch zum europäischen Wettbewerbsrecht und den Binnenmarktfreiheiten der Europäischen Verträge stehen.

\section{Gesundheitsmärkte und EU-Binnenmarkt}

\subsection{Freiheit der Binnenmärkte}

Gesundheitsmärkte sind von allen Binnenmarktfreiheiten betroffen: Freier Personenverkehr, freier Warenverkehr, freier Dienstleistungsverkehr und freier Kapitalverkehr. Der freie Kapitalverkehr kann bei der Privatisierung von Krankenhäusern eine Rolle spielen, der freie Warenverkehr ist bei Medizinprodukten und vor allem Arzneimitteln bereits Realität. Hier gibt es auch eine Vielzahl von europäischen Regulierungen wie Arzneimittelzulassungen und Qualitäts- und Sicherheitsstandards, die den grenzüberschreitenden Handel erleichtern.

Aus der Sicht der Regionen sind von besonderer Bedeutung der freie Personenverkehr von Ärzten und Pflegekräften, die ihren Arbeitsplatz in der EU frei wählen können, die Nachfrage nach Krankenhausleistungen, insbesondere Kuren, und die Nachfrage nach Leistungen von niedergelassenen Ärzten und des Medizinhandwerks, insbesondere in der Zahnprothetik. Aufgrund des starken Einkommensgefälles und der unterschiedlichen Behandlungskosten besteht bei gleichen rechtlichen Rahmenbedingen durch die EU eine ausgeprägte Asymmetrie bei der Mobilität des Angebotes und der Nachfrage zwischen Polen und Deutschland. Dabei geht es in beiden Ländern in erster Linie um die Nachfrager, bei denen die Leistungen durch die Sozialversicherung bezahlt werden, was in Polen alle, 
in Deutschland mehr als neunzig Prozent ${ }^{8}$ der Bürger umfasst, denn nur hier ist ein Marktsegment für die klassischen Gesundheitsdienstleistungen, das hinreichend groß ist, um überhaupt eine Beschäftigungswirkung in ausgewählten Region im EU-Ausland zu entfalten.

Der nationale Sozialversicherungsträger ersetzt bei einer Behandlung im EU-Ausland die Kosten, die im Inland entstanden wären. Damit sind die hohen, von den Versicherten zu tragenden Zuzahlungen für eine Behandlung in Deutschland für polnische Bürger in der Regel prohibitiv, weil das Einkommensniveau und damit die Behandlungskosten in Deutschland so hoch sind, dass der privat zu tragende Anteil die finanziellen Möglichkeiten der Betroffenen in der Regel überschreitet. Eine Wanderung von Patienten von West nach Ost wird wiederum die Ausnahme bleiben, weil die Vorteile aus der Preisdifferen $z^{9}$ nicht die Nachteile einer Behandlung im Ausland kompensieren, weil Gesundheitsgüter Vertrauensgüter mit den oben beschriebenen Eigenschaften und den Konsequenzen für ein überregionales Angebot sind. Zudem ist die ambulante und stationäre Gesundheitsversorgung in Deutschland quantitativ und qualitativ auf einem hohen Niveau, so dass es aus der Sicht deutscher Patienten nur wenige Gründe gibt, sich im Ausland behandeln zu lassen. Das mag sich in der Zukunft in Grenzregionen ändern, so dass hier die Notwendigkeit und Bereitschaft zur Kooperation wachsen könnte.

Deutschland hat seine Sozialgesetzgebung Anfang 2004 den Anforderungen des EU-Rechtes angepasst. Es hat damit die Konsequenzen aus einer ständigen Rechtsprechung des Europäischen Gerichthofes seit 1998 gezogen, die den individuellen Rechten der Patienten, medizinische Leistungen in einem beliebigen Mitgliedsland der EU in Anspruch zu nehmen, einen sehr weitgehenden Vorrang eingeräumt und damit nationale Restriktionen als unvereinbar mit den Binnenmarktfreiheiten erklärt. ${ }^{10}$ Vor 2004 konnten Mitglieder einer deutschen Krankenkasse nur dann im Ausland behandelt werden, wenn es sich um einen Notfall oder einen vorübergehenden Aufenthalt (Urlaub oder Arbeit) handelte, oder wenn die Krankenkasse vorab ihre Zustimmung erklärt hatte, was nur bei sehr speziellen Behandlungen, die es im Inland nicht gab (zum Beispiel spezielle Kuren), erfolgte. Der Genehmigungsvorbehalt ist bei Krankenhausaufenthalten geblieben, weil der Gerichtshof hier zugesteht, dass die Krankenhauskapazitäten vom Staat geplant werden müssen und ein besonderes finanzielles Risiko für den Staat oder die Sozialversicherung besteht. ${ }^{11}$ Aber auch bei stationären Aufenthalten kann die Dienstleistungsfreiheit nicht beliebig eingeschränkt werden, sondern eine Behandlung im Inland muss qualitativ und innerhalb einer angemessenen Wartezeit im Inland möglich sein. Das sind relativ undeutliche Kriterien, die absehbar neue rechtliche Auseinandersetzungen nach sich ziehen werden, aber lange Warteliste oder ein fehlendes Behandlungsangebot im Inland dürften in Zukunft das Recht jedes EU-Bürgers begründen, sich in einem anderen Mitgliedsland behandeln zu lassen.

\subsection{Patientenmobilität}

Formaler Anspruch und tatsächliche Realisierung des Rechtes auf Behandlung im Ausland werden aber davon abhängen, wie hoch der Erstattungsbetrag des nationalen Gesundheitssystems ist, d. h. wie viel ein Patient aus seinem eigenen Einkommen zuzahlen muss. Deshalb wird angesichts der Einkommensdifferenzen zwischen Polen und Deutschland die Inanspruchnahme deutscher Ärzte durch polnische Patienten gering bleiben. Seit Anfang 2004 können deutsche Patienten ambulante Leistungen im EU-Ausland in Anspruch nehmen und sie können, wie bereits vorher, ein ausländisches Krankenhaus oder eine Kureinrichtung, die nach nationalem Recht der Mitgliedslandes anerkannt sind, zur Behandlung wählen, wenn die Krankenkasse das vorher bewilligt (Unveränderte Ausnahme: Notfälle). Daraus könnten sich Hoffnungen und Erwartungen in Polen und anderen mitteleuropäischen Ländern ableiten, dass sie bei Kuren und Maßnahmen der Rehabilitation Patienten aus Deutschland versorgen und so zusätzliche Beschäftigung in Regionen schaffen, die dafür spezielle Angebote haben. Neu ist vor allem, dass die Krankenkassen mit ausländischen Leistungsanbietern Versorgungsverträge abschließen dürfen (§ 140 e SGB V), was allerdings bisher nur in sehr wenigen Fällen stattgefunden hat. Nach meiner Kenntnis gibt es bisher nur einige Beispiele, in denen Betriebskrankenkassen und eine Ersatzkasse Verträge über ambulante Rehabilitation mit einem ausländischen Anbieter abgeschlossen haben. Diese Verträge sind zudem unter sehr kritischer Beobachtung der deutschen Leistungsanbieter und der anderen Krankenkassen, inwieweit die nach deutschem Recht verlangten Qualitätsstandards eingehalten werden. ${ }^{12}$ Nach europäischem Recht dürfen keine identischen Standards verlangt werden, aber die zwischen den Krankenkassen und den ausländischen Anbietern vereinbarte Qualität darf nicht zu stark von den deutschen Regeln abweichen, weil die Kassen in ihren Leistungen an das deutsche Sozialrecht gebunden sind. ${ }^{13}$

Es gibt bereits Erfahrungen mit grenzüberschreitenden Leistungsverträgen zwischen Anbietern und Krankenkassen in Grenzregionen, wo sich Versorgungsangebote ergänzen und insbesondere der Weg ins Krankenhaus oder zu einem Facharzt kürzer ist, wenn man den Weg über die Grenze wählt. ${ }^{14}$ In diesen Regionen fallen auch häufiger Wohn- und Arbeitsort auseinander, so dass es eine besondere Regelung für die Versorgung geben muss. Beispiele gibt es vor allem in der Rhein-Waal-Region zwischen deutschen, belgischen und niederländischen Krankenkassen und Ärzten und Krankenhäusern. Aber auch hier zeigt sich, dass es aus kulturellen und sprachlichen Gründen eine große Treue zu den Ärzten des jeweils eigenen Gesundheitssystems gibt, insbesondere bei der primärärztlichen Versorgung. Vergleichbare Beispiele von grenzüberschreitenden Versorgungsverträgen sind mir an der deutsch-polnischen Grenze nicht bekannt, aber das mag sich ändern, wenn daraus für beide Seiten hinreichend große Vorteile entstehen. ${ }^{15}$ Je spezieller die Krankheit und je notwendiger ein schneller Zugang zum Arzt oder Krankenhaus, desto mehr wird es zu einer gemeinsamen Ver- 
sorgung kommen. Ein polnisch sprechender Arzt, der zur richtigen Zeit die richtige Behandlung durchführt, ist allemal besser als ein deutsch sprechender Arzt, der nicht verfügbar ist - und umgekehrt.

Skeptischer sind die Chancen der polnischen Regionen bei der Erschließung von Märkten für Kuren und Rehabilitationsmaßnahmen zu beurteilen. Neben den oben diskutierten Gründen, das Gesundheitsdienstleistungen lokale Güter sind, kommt noch erschwerend hinzu, dass die Zahl der von den Sozialversicherung finanzierten Kuren und Rehabilitationsmaßnahmen seit Jahren sinkt, weil die Krankenkassen aufgrund ihrer Finanzprobleme an dieser Stelle sparen, aber auch weil der medizinische Sinn bestritten und verstärkt ambulante Rehabilitation angestrebt wird, bzw. im Rahmen einer Integrierten Versorgung Verträge mit Krankenhäuser der Akutversorgung und von Rehabilitationseinrichtungen gemacht werden. Wenn es sich um traditionelle Kurorte der Spitzenklasse wie Karlsbad oder Marienbad handelt, sind die Kostendifferenzen einer Behandlung im Ausland wiederum nicht so groß, dass dies für die Krankenkassen attraktiv ist. So verlangen Kureinrichtungen in Marienbad, Tschechien für drei Wochen stationäre Rehabilitation zwischen $1.900 €$ und $2.200 €$, eine Reha-Klinik in Deutschland verlangt zwischen $2.000 €$ und $2.200 € .{ }^{16}$ Dabei ist auch zu berücksichtigen, dass Kranke in der Regel Wert auf eine Behandlung in der Nähe des Wohnortes legen, weil sie eine lange Anreise scheuen und sicher auch lieber in ihrer Muttersprache angesprochen werden wollen.

Klassische Kurorte im europäischen Ausland konnten von Verträgen mit deutschen Krankenkassen kaum profitieren, was auch mögliche Erwartungen in mitteleuropäischen Regionen relativieren sollte, die traditionell als Orte der Heilung gelten. Optimistischer beurteile ich die Chancen, dass sich traditionelle Urlaubs- oder Kurregionen im Bereich gesundheitsfördernder Angebote, die nicht von den Krankenkassen finanziert werden, sondern die wachsenden Nachfrage im Bereich „Wellness“ befriedigen, einen auch grenzüberschreitenden Markt sichern können. ${ }^{17}$ Die Menschen werden älter, aber es gibt genug Senioren, die Geld ausgeben können und wollen, um bei guter Gesundheit alt zu werden. Gesundheitsfördernde Verhaltsweisen zu erlernen oder den Urlaub mit Sport und Wellness-Angeboten zu kombinieren, ist eine Möglichkeit für Regionen, sich auch international zu profilieren.

Eine weitere Marktchance kann sich für Regionen in Polen, und zwar je näher der westlichen Grenze, desto leichter, in medizinischen Versorgungsbereichen ergeben, wo der Anteil der Zuzahlungen für deutsche Versicherte hoch ist und gleichzeitig eine hoch qualifizierte Arbeit verlangt wird, die in Deutschland deutlich höher als in Polen bezahlt wird. Dazu gehören insbesondere handwerkliche Leistungen wie Zahnprothesen oder andere medizinische Hilfsmittel. ${ }^{18}$ Um hier mehr Sicherheit im Hinblick auf Qualität und Haftung zu schaffen, wäre auch eine europäische Richtlinie zu Dienstleistungen hilfreich, deren Entwurf („Bolkestein-Richtlinie“) von der
Kommission zurückgezogen wurde, weil eine Reihe von Regelungen, von Mitgliedsländern der EU kritisiert wurden.

\subsection{Arbeitsmarktmobilität}

In Deutschland wird die Bevölkerung älter, was in der Zukunft einen größeren Bedarf an Gesundheitsleistungen und vor allem professioneller Pflege erfordern wird. Der Bedarf und das künftige Angebot von Ärzten sind außerordentlich schwer zu prognostizieren, die Prognosen über Pflegebedarfe noch schwieriger, weil sich die Familienstrukturen ändern und künftig weniger Pflege in der Familie erbracht werden könnte. Ein Gutachten im Auftrag des Bundesministerium für Gesundheit und Soziale Sicherung kommt nach Auswertung der einschlägigen Studien zum Ärztemangel zu dem Ergebnis, dass bezogen auf Gesamtdeutschland kein Defizit bei der Versorgung mit Ärzten zu erwarten ist, dass es aber regional und sektoral zu Defiziten kommen wird: In Ostdeutschland werden in Krankenhäusern und bei der hausärztlichen Versorgung Ärzte fehlen. ${ }^{19}$ Es ist absehbar, dass ein Teil des Bedarfs durch Zuwanderung aus Polen gedeckt werden wird, dafür spricht schon die räumliche Nähe, so dass es zu einer weiteren Abwanderung von Ärzten und Pflegekräften aus Polen nach Deutschland kommen wird. Zumindest ist das zu erwarten, wenn die bestehenden Einkommensdifferenzen fortdauern. ${ }^{20}$ Nach einer Meldung des "Spiegel“ vom 2. Mai 2005 ist allein die Zahl der Ärzte, die in Ostdeutschland arbeiten und aus Polen kommen, von 67 in 2001 auf 376 in 2004 gestiegen. Das halte ich für keine wünschenswerte Entwicklung, weil Deutschland dann seine Versorgungsprobleme nach Polen exportiert und auch in Polen wie in Deutschland am stärksten die ländlichen Regionen betroffen sein werden. Bei allen Chancen durch die EU, hier liegen Risiken für Polen, denn die Mobilität von Arbeitnehmern und Selbständigen wird die Europäische Union auf Dauer wegen der in den Europäischen Verträgen vereinbarten Binnenmarktfreiheiten weder verhindern können noch wollen.

\section{Fazit}

Es gibt eine gespaltene Mobilität auf Gesundheitsmärkten, deshalb sind Risiken und Chancen ungleich zwischen Polen und Deutschland verteilt.

1. Gesundheitsdienstleistungen sind lokale Güter und die Chancen von Regionen sind gering, ihre Leistungen international anzubieten.

2. Ärzte und Pflegekräfte sind mobil und werden zumindest zum Teil ihre Chancen nutzen, in einem gemeinsamen Binnenmarkt dort zu arbeiten, wo sie höhere Einkommen erzielen.

3. Geld allein ist sicher nicht das einzige Motiv eines Menschen, aber doch ein starkes. Gerade wenn Grenzen immer weniger wichtig werden, werden die ökonomischen Hebel stärker wirken. 


\section{Literatur}

Deutsches Institut für Wirtschaftsforschung (2001), Endbericht des Gutachtens im Auftrag des Bundesministeriums für Wirtschaft und Technologie „Wirtschaftliche Aspekte der Märkte für Gesundheitsdienstleistungen. Ökonomische Chancen unter sich verändernden demographischen und wettbewerblichen Bedingungen in der Europäischen Union, Berlin www.diw.de/deutsch/produkte/publikationen/gutachten/docs/diw_GesundheitsDL_200112.pdf (16. 8. 2005)

Eichenhofer, Eberhard (2003), Rechtliche Rahmenbedingungen für grenzüberschreitende Gesundheitsleistungen im europäischen Binnenmarkt, in: Klusen, Norbert, Hrsg., Seiten 145-160

Hajen, Leonhard; Paetow, Holger; Schumacher, Harald (2004), Gesundheitsökonomie, 2. Aufl., Stuttgart

Heese, Jürgen (2005), Praxisbericht: Verträge mit ausländischen Leistungserbringern, in: AOK-Bundesverband, Europa für die Versicherten gestalten, Bonn, Seiten 62-68

Klusen, Norbert, Hrsg. (2003), Europäischer Binnenmarkt und Wettbewerb Zukunftsszenarien für die GKV, Baden-Baden

Reichelt, Peter; Agasi, Susanne (2003), Die Zukunft der grenzüberschreitenden Gesundheitsversorgung aus der Perspektive der Versicherten, in: Klusen, Norbert, Hrsg., Seiten 193-210

Rice, Thomas (2004), Stichwort: Gesundheitsökonomie, Eine kritische Auseinandersetzung, Bonn; Original: The Economics of Health Reconsidered, Chicago 1998

Romboll-Management (2004), Gutachten im Auftrag des Bundesministeriums für Gesundheit und Soziale Sicherung zum „Ausstieg aus der kurativen ärztlichen Berufstätigkeit in Deutschland“, Abschlussbericht, Hamburg, www.bmgs.bund.de/download/broschueren/F335.pdf (16. 8. 2005)

Rydzewski, Marek (2005), Gute Versorgung wächst mit gegenseitigem Verständnis, in: Gesundheit+Gesellschaft, 8. Jg., Ausgabe 4/05, Seite 31

Schaub, Vanessa Elisabeth (2000), Grenzüberschreitende Gesundheitsversorgung in der Europäischen Union, Baden-Baden

Turner, Adair (2002), Just Capital, The Liberal Economy, London, Basingstoke, Oxford

WHO (1999), European Observatory on Health Care Systems, Health Care Systems in Transition, Poland, Copenhagen

Wille, Eberhard (2005), Auswirkungen der europäischen Integrationspolitik auf das deutsche Gesundheitswesen, in: AOK-Bundesverband, Europa für die Versicherten gestalten, Bonn, 29ff

\section{Fußnoten}

1 Überarbeitete Fassung eines Referates auf der Konferenz der NikolausKopernikus-Universität, Thorn zu „Globalisierung und Regionen in der Europäischen Union“ am 19.9.2005

2 Turner, 2002, 31ff

3 DIW, 2001, 51ff

4 Hajen, Paetow, Schumacher, 2004, 40ff

5 In Deutschland verpflichtet das Sozialgesetzbuch die Leistungsanbieter zur Qualitätssicherung (§ 135 a SGB V und § 20 SGB IX). Für Rehabi- litationseinrichtungen haben sich beispielsweise die Spitzenverbände der Krankenkassen auf ein gemeinsames Qualitätssicherungsprogramm verständigt (www.qs-reha.de (16.8.2005) )

$6 \mathrm{Zu}$ der falschen Analogie des Wettbewerbsmarktes vgl. Rice, 2004, 31ff

7 Reichelt, Agasi, 2003, 200

8 Die nicht in den gesetzlichen Krankenkassen versicherten Bürger sind Teil einer Sonderversorgung (Beamte, Soldaten) oder bis auf einen kleine Gruppe in einer privaten Krankenversicherung.

9 Ein Vergleich der Preise von Kureinrichtungen in Polen, Tschechien und der Slowakei zeigt, dass zwischen und auch innerhalb dieser Länder große Preisunterschiede für eine dreiwöchige Kur bestehen, aber Kuren im Vergleich zu Deutschland um mehr als ein Drittel billiger sind. Geprüft wurden Angebote im Katalog oder im Internet, was einen Vergleich der Qualität nur begrenzt ermöglicht. Der Vergleich wurde von Katarzyna Janul erstellt und ist bei mir abrufbar: Leonhard.Hajen@wiso.uni-hamburg.de

10 Eichenhofer, 2003, 145ff

11 EuGH, Rs C-157/99, Slg. 2001, S. I-5473, Rn 76

12 Eine Recherche auf der Basis des Internets für ausgewählte, osteuropäische Kurorte zeigt, dass Angaben zur Qualitätssicherung die seltene Ausnahme sind.

13 Der Konflikt hat auch die politische Ebene des Bundestages erreicht: vgl. die Frage des Abgeordneten Ernst Burgbacher (FDP) und die Antwort von Staatssekretär Dr. Klaus Theo Schröder vom 22. 4. 2005, Bundestagsdrucksache 15/5414

14 Schaub, 2000, 79ff

15 Rydzewski , 2003, 31 berichtet, dass sich die Zusammenarbeit zwischen der AOK, einer großen Krankenkasse in der Region, und dem polnischen Sozialversicherungsträger NFZ zur Zeit auf den Austausch von Informationen beschränkt.

16 Information der Barmer Ersatzkasse vom 21. 4. 2005

17 Beispielsweise hat das Sanatorium „Pod Tezniami“ in dem polnischen Kurort Ciechocinek $40 \%$ Gäste aus Deutschland. Nach Auskunft des Sanatoriums sind die Gäste Selbstzahler.

18 So informiert die AOK-Brandenburg ihre Mitglieder über die Möglichkeiten, sich im Nachbarland Polen behandeln zu lassen und verweist auf sieben Praxen in der Grenzregion, mit denen vereinfachte Formalitäten vereinbart wurden. Vgl. Märkische Oderzeitung vom 15. 9. 2005: Steit um die „Dritten“ aus Polen

19 Romboll-Management, 2004, 63ff

20 Vergleichbare Einkommensdaten für Ärzte und Pflegekräfte in Deutschland und Polen liegen in den Statistiken der Europäischen Union, der OECD, der EU und WHO nicht vor. Es dürfte für Polen auch methodisch sehr schwer zu ermitteln sein, weil ca. ein Drittel des Einkommens der Ärzte aus privaten Zuzahlungen besteht, die neben den offiziellen Einkommen erzielt werden; das Grundeinkommen verdoppelt sich im Durchschnitt durch Bereitschaftsdienste und Überstundenzuschläge (WHO, 1999, S. 17f und 37). Die Zeitung Gazeta Prawna berichtet am 15. 3. 05 über ein durchschnittliches Einkommen der polnischen Ärzte von 500€, was aber nur das offizielle Grundeinkommen umfassen dürfte (http://www.jobmed.pl/artykul.php?idartykul rodzaj=1\&idartykul=2028 (4. 9. 2005)). 